\title{
Was folgt auf die Millenniums-Entwicklungsziele?
}

\section{Einleitung}

Das Jahr 2015 kann ein Meilenstein für globale Entwicklung werden. Die Staaten handeln neue Ziele für nachhaltige Entwicklung (Sustainable Development Goals, SDGs) aus, die die 2015 auslaufenden Millenniumentwicklungsziele (Millennium Development Goals, MDGs) ablösen sollen. Die SDGs könnten als politische Zielsetzungen und Kompass der Weltgemeinschaft eine zentrale Bedeutung für die Zukunft globaler Entwicklung und die Transformation in Richtung ökonomischer, sozialer und ökologischer Nachhaltigkeit erlangen. Im Gegensatz zu den MDGs, die auf die menschliche Entwicklung in Entwicklungsländern abzielten, sollen die SDGs alle Dimensionen nachhaltiger Entwicklung adressieren und für alle Staaten gültig sein. Auf der UN-Konferenz über Nachhaltige Entwicklung (UNCSD, „Rio+20-Konferenz“) im Jahr 2012 wurde beschlossen, die SDGs zu erarbeiten. Im Sommer 2014 hat die Offene Arbeitsgruppe (Open Working Group, OWG) der Vereinten Nationen (VN) zu den SDGs einen Vorschlag für 17 Entwicklungsziele für die Post-2015-Agenda vorgelegt. Derzeit laufen, darauf aufbauend, die Verhandlungen der VN-Mitgliedsstaaten. Das Ziel ist, im September 2015 die SDGs verabschieden zu können.

Die 17 von der OWG vorgeschlagenen SDGs stellen einen Katalog wichtiger Prioritäten für nachhaltige Entwicklung dar. Eine grundlegende Dimension, so argumentieren wir in diesem Beitrag, ist jedoch nicht ausreichend berücksichtigt: die Notwendigkeit, die Erdsystemleistungen zu schützen, die unabdingbar sind, um die Zukunft menschlicher Entwicklung gewährleisten zu können. Die SDGs müssen die „Leitplanken“ (WBGU 2014) des Erdsystems einbeziehen, die den begrenzten Naturraum beschreiben, innerhalb dessen bald neun Milliarden Menschen Armutsreduzierung und Wohlfahrtsziele realisieren können. Werden diese planetarischen Leitplanken überschritten, würden die Lebensbedingungen der derzeit vulnerabelsten zwei Milliarden Menschen weiter unterminiert und Existenzgrundlagen kommender Generationen gefährdet. Planetare Leitplanken zum Schutz des Erdsystems, dem umfassendsten globalen Gemeinschaftsgut der 
Menschheit, sollten ein zentrales Element der Post-2015-Entwicklungsagenda werden.

Im Folgenden umreißen wir zunächst den Entstehungskontext sowie die Erfolge und Grenzen der Millenniums-Entwicklungsstrategie. Anschließend argumentieren und erläutern wir, warum und wie die Post-2015-Agenda die globalen Umweltveränderungen berücksichtigen sollte, bevor wir in den Schlussbemerkungen einen Ausblick auf wichtige Prioritäten der globalen Kooperation und auf Forschungsaktivitäten in diesem Kontext geben.

\section{Die Millenniums-Entwicklungsstrategie entsteht im Kontext der Über- windung des Kalten Krieges}

Die Millenniums-Entwicklungsstrategie entstand in der letzten Dekade des 20. Jahrhundert. Nach dem Ende des Kalten Krieges kam es in der Periode zwischen 1990 und 2000 zu einer paradoxen Doppelbewegung: Auf der einen Seite sanken die öffentlichen Investitionen in die Entwicklungszusammenarbeit weltweit. Ohne die Systemkonkurrenz mit der Sowjetunion schien in den westlichen Ländern ein wichtiger Anreiz der Kooperation mit den Ländern des Südens entfallen zu sein. Kurzfristige Interessenskalküle führten zu einer Reduzierung der Bedeutung der Entwicklungspolitik, die eben nicht nur auf die Überwindung von Entwicklungsblockaden in den Entwicklungsländern ausgerichtet war, sondern zugleich auch ein Instrument des Kalten Krieges darstellte, das nach dessen Ende an Relevanz verlor.

Zugleich waren die 1990er Jahre jedoch auch die Dekade, in der infolge der unerwarteten Überwindung des Kalten Krieges international intensiv über „Friedensdividenden“ und „Weltinnenpolitik“ diskutiert wurde. Die 1990er Jahre waren die Dekade der großen VN-Weltkonferenzen, in deren Kontext sich die Staaten und zunehmend auch zivilgesellschaftliche Organisationen mit den Menschheitsfragen des 21. Jahrhunderts auseinandersetzten. 1990 fand der Weltkindergipfel in New York statt; 1992 die Weltkonferenz zu Umwelt und Entwicklung in Rio de Janeiro; 1993 die Weltmenschenrechtskonferenz in Wien; 1994 die Konferenz zu Bevölkerung und Entwicklung; 1995 der Weltgipfel zu Sozialer Entwicklung in Kopenhagen; im gleichen Jahr die Weltfrauenkonferenz in Peking; schließlich 1997 der Städtegipfel in Istanbul.

Die MDGs können als ein Ergebnis dieser widersprüchlichen Doppelbewegung erachtet werden. Das Ende des Kalten Krieges schaffte Freiräume, um im Rahmen der Weltkonferenzen über gemeinsame globale Herausforderungen nachzudenken. Diese Dynamiken mündeten in einer erneuerten Legitimationsgrundlage 
Was folgt auf die Millenniums-Entwicklungsziele?

für die internationale Entwicklungskooperation. Die Entwicklungspolitik entkoppelte ihre Existenzberechtigung von der Systemkonkurrenz mit der kommunistischen Welt und basierte ihre Legitimation nun auf den Herausforderungen einer interdependenten, zusammenwachsenden Weltgesellschaft, deren Konturen während der Weltkonferenzen vorgedacht worden waren (Kennedy/Messner/ Nuscheler 2001).

In diesem internationalen Milieu beschlossen die VN-Mitgliedsstaaten auf dem Millennium-Gipfel in New York im Jahr 2000 die Millenniumserklärung, die als Grundlage für die Erarbeitung der MDGs diente. Die MDGs umfassten acht Entwicklungsziele, zu deren Erreichung bis 2015 sich die internationale Gemeinschaft verpflichtet hat und in deren Zentrum die Überwindung von Armut steht. Lediglich MDG 7 zielt auf Umweltschutz und die nachhaltige Nutzung natürlicher Ressourcen, ist jedoch eng, schwach und unpräzise formuliert. Die MDGs prägten als ,globales Narrativ“ die Diskurse der internationalen Entwicklungspolitik und gaben ihr eine klare und verständliche Richtung. Sie trugen auch dazu bei, den Trend rückläufiger Entwicklungshilfe zu beenden und ein Zielsystem vorzugeben, das den Schwerpunkt auf Armutsbekämpfung und Investitionen in die essenziellen Grundlagen menschlicher Entwicklung legte, beispielsweise Gesundheit und Grundbildung.

Die MDGs sind insofern ein Ergebnis des Endes des Kalten Krieges und insbesondere der Weltkonferenzen, doch sie „halbierten“ die Erkenntnisse und Lehren ebendieser Weltkonferenzen, denn diese hatten gezeigt, dass zur Lösung der weltumspannenden Entwicklungs-, Umwelt- und Sicherheitsprobleme Veränderungsprozesse in Industrie- und Entwicklungsländern sowie im internationalen System gleichermaßen notwendig wären. Das Abschlussdokument der Rio-Konferenz zu Umwelt und Entwicklung von 1992, die viel zitierte Agenda 21, hatte diese Perspektive bereits vorgegeben. Die folgenden Weltkonferenzen bestätigten diesen Lernprozess (Messner/Nuscheler 1998). Doch mit den MDGs wurden Ziele formuliert, die sich ausschließlich an die Entwicklungsländer richteten. Veränderung wurde also von den Akteuren des Südens erwartet. Sie sollten durch Entwicklungsinvestitionen des Nordens unterstützt werden. Die internationale Entwicklungskooperation blieb dem etablierten Geber-Nehmer-, Nord-Süd-Schema verhaftet. Ziele, an denen sich die Industrieländer im Prozess ihrer eigenen Entwicklung hätten orientieren können oder aus der Perspektive gemeinsamer, globaler Verantwortung hätten orientieren sollen, wurden nicht verabredet. 


\section{Das beginnende 21. Jahrhundert: Machttransformationen und der Übergang zum Anthropozän}

Im Verlauf der ersten Dekade des 21. Jahrhunderts wurden große Hürden für globale Kooperation und erfolgreichen Multilateralismus deutlich. Gleichzeit wurde immer offensichtlicher, was während der Weltkonferenzen der 1990er Jahre bereits diskutiert, aber im Zielsystem der MDGs zunächst ausgeblendet worden war, dass nämlich den globalen Herausforderungen, zum Beispiel dem Klimawandel oder den Risiken weltweit verflochtener Finanzmärkte, nur durch reziproke Formen der Kooperation zwischen und Veränderungsprozessen in Industrie-, Schwellen- und Entwicklungsländern sowie neuen Formen der Global Governance begegnet werden kann.

Ein wichtiger Grund für neue Herausforderungen für den Multilateralismus ist der rasante Aufstieg zahlreicher Entwicklung- und Schwellenländer wie China, Indien, Brasilien, Südafrika und Indonesien sowie die sich dadurch auf globaler Ebene verschiebenden wirtschaftlichen und politischen Gewichte und damit einhergehende Machtverlagerungen- und -blockaden (Kaplinsky/Messner 2008; Kupchan 2012) - eine der entscheidenden Wellen des globalen Wandels (Messner 2011). Diese neuen Machtkonstellationen waren im Verlauf der Weltkonferenzen der 1990er Jahre noch nicht absehbar gewesen. Die optimistische Sicht der Dinge betont, dass eine größere Rolle der Schwellen- und Entwicklungsländer dazu beitragen kann, dass Global Governance potenziell demokratischer und inklusiver wird. Die pessimistische Sichtweise auf die zunehmend multi- oder auch non-polare Welt betont die Schwierigkeiten globaler Kooperation im Kontext tektonischer Machtverschiebungen (Bremmer 2012; Kupchan 2012): Die Klimaverhandlungen beispielsweise stocken und die aktuelle Welthandelsrunde macht kaum Fortschritte.

Sowohl im Rahmen der VN-Konferenzdiplomatie als auch in der multilateralen Welthandelsorganisation dominiert oft eine konfrontative Nord-Süd-Dichotomie, die Einigungen schwierig macht. Ein wichtiges Beispiel dafür war das enttäuschende Scheitern der Weltklimakonferenz in Kopenhagen im Jahr 2009. Unterschiedliche Ansätze der Kooperationsforschung liefern hier wertvolle Erkenntnisse. Polyzentrische Machtkonstellationen und -spiele können globale Kooperation behindern, insbesondere wenn Basismechanismen von Kooperation (wie Vertrauensbeziehungen, dichte Kommunikationsnetze, gemeinsame Vorstellungen von Fairness, Reziprozität) nur unzureichend entwickelt sind. Dies ist insbesondere dort der Fall, wo sich Akteure gegenüberstehen, die bisher kaum gemeinsame Ko- 
operationserfahrungen aufbauen konnten - z. B. im Rahmen der G 20 (Messner/ Guarín/Haun 2013).

Gleichzeitig zeigt die Nachhaltigkeitsforschung seit Beginn des neuen Jahrtausends immer deutlicher, dass die MDGs sowie menschliche Entwicklung für eine bald neun Milliarden Menschen umfassende Zivilisation ohne die Berücksichtigung globaler Umweltveränderungen nicht auf Dauer realisierbar sind. Durch den verengten Fokus auf soziökonomische Entwicklung und das mangelnde Augenmerk auf ökologische Nachhaltigkeit stößt die Entwicklung der Weltwirtschaft an die Grenzen des Erdsystems. Die Nachhaltigkeitsforschung untermauert dies: Um die Basis für nachhaltige Entwicklung zu schützen, müssen die "planetarischen Grenzen“" (planetary boundaries) (Rockström u. a. 2009 a, 2009 b; Steffen u. a. 2015) bzw. die vom Wissenschaftlichen Beirat für Globale Umweltveränderungen seit Mitte der 1990er Jahre entwickelten „planetarischen Leitplanken“, das heißt quantitativ definierbare Schadensgrenzen, jenseits derer das Erdsystem durch anthropogene globale Umweltveränderungen so stark verändert wird, dass die Folgen gesellschaftlich intolerabel wären (WBGU 2011), respektiert werden. ${ }^{1}$

Während sich die menschliche Zivilisation innerhalb der letzten mehr als 11.000 Jahre während des Holozäns entwickelt hat (Walker u. a. 2009), einer erdgeschichtlichen Phase mit vergleichsweise stabilen Umweltbedingungen, bedeutet ein Überschreiten der planetarischen Grenzen und ein Verlassen dieses ,safe operating space" (Rockström u. a. 2009 b) hohe Risiken für die Menschheit. Laut zahlreicher Wissenschaftler hat die Menschheit mittlerweile so starken Einfluss auf das Erdsystem genommen, dass wir davon ausgehen müssen, dass wir inzwischen tatsächlich in einer neuen geologischen Epoche leben, dem Anthropozän (Crutzen 2000; Steffen u. a. 2011), das Erdzeitalter der Menschen, die zur treibenden Kraft im Erdsystem geworden sind. Durch das Durchbrechen der planetarischen Grenzen oder Leitplanken können entweder „Kipppunkte des Erdsystems“ (Lenton u. a. 2007) überschritten werden, sodass nicht-lineare, abrupte Änderungen ausgelöst werden, oder schleichende, fortdauernde Veränderungen über lange Zeitspannen so stark kumulieren, dass sie ein Ausmaß erreichen, das nicht mehr

1 Der Wissenschaftliche Beirat für Globale Umweltveränderungen (WBGU) hat zunächst die Leitplanken für anthropogenen Klimawandel quantifiziert (WBGU 1995), später dann auch für Umweltherausforderungen wie Landdegradation, Verlust der Artenvielfalt und Ozeanversauerung. Das Konzept der planetarischen Grenzen (planetary boundaries), vorgeschlagen von Rockström und seinen Kollegen (Rockström u. a. 2009 a, b), ist dem WBGU-Konzept der Leitplanken sehr ähnlich, und es lassen sich daraus für die SDGs und für die globale Governance dieselben Schlussfolgerungen ziehen. Dirk Messner ist Co-Vorsitzender des WBGU, Clara Brandi wissenschaftliche Referentin des WBGU. 
tolerabel ist (WBGU 2014) - und die Basis für zukünftige Entwicklung gefährden (Messner/Rahmstorf 2010).

\section{Die nächste Generation globaler Entwicklungsziele - Prioritäten und Herausforderungen}

Die Festlegung globaler Ziele für nachhaltige Entwicklung ist ohne das Einbeziehen planetarischer Leitplanken nicht mehr seriös möglich, da globale Umweltveränderungen, wie z. B. der Klimawandel, der Verlust biologischer Vielfalt und weltweite Bodendegradation, erhebliche Risiken bergen. Das Durchbrechen der Leitplanken muss verhindert werden, denn dies gefährdet die natürlichen Lebensgrundlagen der Menschheit und damit auch die Grundlage für menschliche Entwicklung.

Da das Einhalten der Leitplanken die Voraussetzung ist, um die Grundlage für menschliche Entwicklung langfristig zu sichern, ist der Verbleib innerhalb der Leitplanken die Basis für die Realisierung der SDGs insgesamt. Der SDG-Katalog sollte deshalb das Ziel "Sicherung der Erdsystemleistungen“ beinhalten. Wie kürzlich vom WBGU (2014) vorgeschlagen, sollte ein solches Ziel die folgenden sechs Leitplanken des Erdsystems adressieren: (1) die Erwärmung des Klimasystems auf $2^{\circ} \mathrm{C}$ und (2) die Ozeanversauerung auf den $\mathrm{pH}$-Wert 0,2 begrenzen (durch den Stopp globaler $\mathrm{CO}_{2}$-Emissionen aus fossilen Quellen bis 2070); (3) anthropogene Treiber für den Verlust von biologischer Vielfalt und Ökosystemleistungen bis 2050 stoppen; (4) Netto-Land- und Bodendegradation bis 2030 stoppen; (5) die Gefährdung durch langlebige anthropogene Schadstoffe bis 2050 stoppen, z. B. Quecksilber, Plastik, spaltbares Material; und (6) den Verlust von nicht rückgewinnbarem Phosphor als essenzielle Ressource für Landwirtschaft und Ernährungssicherheit bis 2050 stoppen.

Es gibt bereits erste Überlegungen für institutionelle Reformprozesse, die die Verankerung der Erdsystemperspektive in der Global Governance begünstigen würden. ${ }^{2}$ Ein Vorschlag lautet, dass sich die VN-Vertragsstaaten darauf einigen, im Rahmen der relevanten VN-Umweltkonventionen die Umsetzung des Erdystem-SDGs zu verhandeln und sowohl Monitoring- als auch Review-Prozesse für die nationale Ausgestaltung und Implementierung des SDGs umzusetzen (WBGU 2014).

Obwohl einige Stimmen dafür plädieren, die planetarischen Grenzen oder Leitplanken in die SDGs zu integrieren (Griggs et al. 2013, Kim et al. 2013, Rock-

2 So beispielsweise Biermann u. a. 2012, Bosselmann u. a. 2012, Kim/Bosselmann 2013, Galaz u. a. 2012, Rockström u. a. 2013, Steffen u. a. 2011 und WBGU 2014. 
Was folgt auf die Millenniums-Entwicklungsziele?

ström et al. 2013, Raworth 2014, WBGU 2014, Brandi/Messner 2015), gibt es große Hürden für ehrgeizige SDGs, die die Erdsystemperspektive berücksichtigen (WBGU 2014). Zum einen stellen sich viele OECD-Länder sowie aufstrebende Mächte gegen einen neuen Zielkatalog, der weit über Armutsbekämpfung hinaus geht und auch ihnen selbst ambitionierte Ziele auferlegt. Zum anderen missinterpretierten einige Akteure (z. B. in den Entwicklungsländern) die planetarischen Grenzen oder Leitplanken als Begrenzung ihrer zukünftigen Entwicklungschancen. Dabei stellen planetarische Leitplanken, wie der WBGU gezeigt hat, keine Begrenzung für die Entwicklung der Ärmsten dar. Das Einhalten der Leitplanken ist vielmehr eine unabdingbare Voraussetzung für Armutsbekämpfung und Entwicklung (WBGU 2014). Es sind vor allem die Konsum- und Lebensstilentscheidungen der globalen Mittel- und Oberklassen und deren ökologischer Fußabdruck, die das Erdsystem am stärksten unter Druck setzen.

Doch trotz ihrer fundamentalen Bedeutung für globale Entwicklung werden weder die planetarischen Grenzen noch die Leitplanken explizit im OWG-Vorschlag erwähnt (OWG 2014). Aus der Erdystemperspektive sind die von der OWG vorgeschlagenen SDGs daher nicht ambitioniert genug. Lediglich drei der 17 OWG-SDGs erwähnen Umweltveränderungen, die für das Einhalten der Leitplanken relevant sind (Brandi/Messner 2015): Zum einen enthält der aktuelle SDG-Vorschlag ein Klimaziel. Das SDG 13 nennt jedoch weder die $2^{\circ} \mathrm{C}$-Leitplanke noch andere relevante Grenzwerte. Das SDG 14 legt das Augenmerk auf die Ozeane, Meere und Meeresressourcen und erwähnt die leitplankenrelevante Ozeanversauerung, beinhaltet jedoch ebenfalls keine operationalisierbaren Grenzwerte. Das SDG 15, das sich dem Schutz terrestrischer Ökosysteme und dem Erhalt der Artenvielfalt widmet, ist ebenfalls nicht umfassend und ehrgeizig genug formuliert, um das Einhalten der planetarischen Leitplanken zu gewährleisten. Darüber hinaus enthält die Liste der OWG weitere SDG- und Target-Vorschläge mit Umweltbezug, beispielsweise das SDG 7, in dessen Fokus unter anderem der Ausbau erneuerbarer Energie steht, oder SDG 12, das auf nachhaltige Konsumund Produktionsmuster zielt. Aber auch diese Ziele sind nicht so formuliert, dass sie den Verbleib innerhalb der planetarischen Leitplanken oder die Sicherung der relevanten Erdsystemleistungen sicherstellen können (Brandi 2015).

Der Ende 2014 vorgelegte Synthesebericht von VN-Generalsekretär Ban Kimoon erwähnt zumindest die ,planetary boundaries“ (Ki-moon 2014). Er wiederholt die 17 SDG-Vorschläge der OWG, geht aber unter der Überschrift „Planet“ ein Stück weit darüber hinaus: Ban Ki-moon schreibt, dass die planetarischen Grenzen respektiert werden müssen und erwähnt die $2^{\circ} \mathrm{C}$-Leitplanke der internationalen Klimapolitik. Doch durch das schlichte Wiederholen der 17-OWG-Vor- 
schläge bietet der Synthesebericht letztlich keinen geeigneten Rahmen, um die wichtigen Erdsystemleistungen langfristig zu bewahren und die Zukunft globaler Entwicklung sichern zu können.

Derzeit liegt der Ball bei den VN-Vertragsstaaten: Bis September 2015 verhandeln sie über die Post-2015-Agenda, die im Rahmen der dann stattfindenden VNGeneralversammlung angenommen werden soll. Die Gestaltung und Umsetzung der Post-2015-Agenda wird mit darüber entscheiden, ob es der Menschheit gelingen wird, Verantwortung für die Stabilität des Erdsystems und damit die Existenzgrundlagen vieler kommender Generationen zu übernehmen (Messner 2012). Auch wenn es gute Gründe dafür gibt, das aktuelle Paket der OWG-Vorschläge nicht erneut aufzuschnüren und damit dessen Verabschiedung zu gefährden, können die Leitplanken stärker in den SDGs berücksichtigt werden, sowohl in der Präambel als auch auf der Ebene der Unterziele (,,targets“) und Indikatoren. Um die zukünftige Basis für globale Entwicklung zu bewahren, sollte sich die internationale Gemeinschaft in diesen Verhandlungen dafür stark machen, ein umfassendes Erdsystem-SDG in der Post-2015 Agenda zu verankern.

\section{Schlussbemerkungen}

Mit Blick auf die Zukunft der internationalen Beziehungen zeigt unsere Bestandsaufnahme: Die Erdsystemsicht sollte stärker in den SDGs verankert werden. Die Erdsystemperspektive unterstreicht dabei, dass die SDGs ,universal“ gelten müssen, da nur so die planetarischen Leitplanken eingehalten werden können: Es geht um die Einigung auf einen Katalog von Zielen nachhaltiger Entwicklung, der für alle Staaten gilt - für OECD genauso wie für Nicht-OECD-Länder. Nur auf diesem Wege kann der ,safe operating space“ für die Menschheit, und für Armutsbekämpfung und Entwicklung, bewahrt werden. Die von der VN-Generalversammlung angenommenen SDGs sollten - und werden voraussichtlich - deshalb auch für Länder wie die USA und die Staaten der EU gelten. Dies ist im Vergleich mit den MDGs, die sich nur an die Entwicklungsländer richteten, ein wichtiger Paradigmenwechsel, der an die Erkenntnisse der Weltkonferenzen der 1990er Jahre anknüpft, in denen die gemeinsame Veränderungsbereitschaft von Industrie-, Entwicklungs- und Schwellenländern bereits angemahnt worden war.

Auch jenseits der Post-2015-Agenda sollte die Erdsystemsicht in der globalen Kooperation eine wichtigere Rolle spielen. Darüber hinaus sollte die Erdsystemperspektive ebenfalls in der wissenschaftlichen Auseinandersetzung eine zentrale Rolle spielen. Vor allem sollte die zunächst naturwissenschaftlich orientierte Erdsystemperspektive stärker in die sozialwissenschaftliche Forschung eingebettet 
Was folgt auf die Millenniums-Entwicklungsziele?

werden, nicht zuletzt in die Disziplin der Internationalen Beziehungen. Für eine stärkere Verankerung der Erdsystemperspektive in der Forschung zu Global Governance gibt es bereits erste gute Anknüpfungspunkte (Biermann 2014; WBGU 2011; Messner 2015). Die Erdsystemperspektive unterstreicht somit die Bedeutung nicht nur transnationaler, sondern auch transdisziplinärer Forschungskooperationen.

\section{Literatur}

Biermann, Frank, 2014: Earth System Governance. World Politics in the Anthropocene, Cambridge, MA.

Biermann, Frank/Abbott, Kenneth/Andresen, Steinar/Bäckstrand, Karin/Bernstein, Steven/Betsill, Michele M./Bulkeley, Harriet u. a., 2012: Navigating the Anthropocene: Improving Earth System Governance, in: Science 335, 1306-1307.

Bosselmann, Klaus/Brown, PeterG./Mackey, Brendon, 2012: Enabling a Flourishing Earth: Challenges for the Green Economy, Opportunities for Global Governance, in: Review of European Community and International Environmental Law 21, 23-30.

Brandi, Clara, 2015: Safeguarding the Earth System as a Priority for Sustainable Development and Global Ethics, in: Journal of Global Ethics (im Erscheinen).

Brandi, Clara/Messner, Dirk, 2015: Die Herausforderung für 2015: Globale Entwicklung innerhalb planetarischer Leitplanken sichern, Die aktuelle Kolumne, 09.02.2015, Bonn: Deutsches Institut für Entwicklungspolitik (DIE).

Bremmer, Ian, 2012: Every Nation for Itself: Winners and Losers in a G-Zero World, New York.

Crutzen, Paul, 2000: The Anthropocene, in: The Global Change Newsletter (41), 17-18.

Galaz, Victor/Biermann, Frank/Crona, Beatrice/Loorbach, Derk/Folke, Carl/ Olsson, Per/Nilsson, Måns u. a., 2012: Planetary Boundaries - Exploring the Challenges for Global Environmental Governance. Current Opinion in Environmental Sustainability, in: Current Opinion in Environmental Sustainability 4, 80-87.

Griggs, David/Stafford-Smith, Mark/Gaffney, Owen/Rockström, Johan/Öhman, Marcus C./Shyamsundar, Priya/Steffen, Will u. a., 2013: Sustainable Development Goals for People and Planet, in: Nature 495, 305-307.

Kaplinsky, Raphael/Messner, Dirk, 2008: The Impact of Asian Drivers on the Developing World, in: World Development 36 (2), 197-209. 
Kennedy, Paul/Messner, Dirk/Nuscheler, Franz, 2001: Global Trends and Global Governance, London.

Kim, Rakhyun E./Bosselmann, Klaus/Mauerhofer, Volker, 2013: Planetary Boundaries in Post-2015 Sustainable Development Goals: Safeguarding Ecological Integrity as a Priority Goal and a Grundnorm of International Law, http://planetaryboundariesinitiative.org/wp-content/uploads/2013/07/TheKim-Report-September-2013.pdf (Stand: 02.02.15).

Kim, Rakhyun.E./Bosselmann, Klaus, 2013: International Environmental Law in the Anthropocene: Towards a Purposive System of Multilateral Environmental Agreements, in: Transnational Environmental Law, 1-25.

Ki-moon, Ban, 2014: The Road to Dignity by 2030: Ending Poverty, Transforming All Lives and Protecting the Planet, Synthesis Report of the SecretaryGeneral on the Post-2015 Sustainable Development Agenda, New York.

Kupchan, Charles, 2012: No One's World: The West, The Rising Rest, And The Coming Global Turn, Oxford.

Lenton, Timothy M./Held, Hermann/Kriegler, Elmar/Hall, Jim W./Lucht, Wolfgang/Rahmstorf, Stefan/Schellnhuber, John, 2007: Tipping Elements in the Earth's Climate System. Proceedings of the National Academy of Sciences (PNAS) 105 (6), 1786-1793.

Messner, Dirk, 2011: Three Waves of Global Change: the Dynamics of Global Governance in the first Half of the 21st Century, in: Thomas Fues/Liu Youfa (Hrsg.), Global Governance and Building a harmonious World: A Comparison of European and Chinese Concepts for International Affairs, Bonn, 9-38.

Messner, Dirk, 2012: Globale Ressourcenknappheiten und Erdsystemgrenzen im Anthropozän. Treiber, Lösungsansätze und Ambitionsniveaus der Transformation zur Nachhaltigkeit, in: Michael Reder/Hanna Pfeifer (Hrsg.), Kampf um Ressourcen. Weltordnung zwischen Konkurrenz und Kooperation, Stuttgart, 138-158.

Messner, Dirk, 2015: Deutschland als Gestaltungsmacht in der globalen Nachhaltigkeitspolitik: Chancen und Herausforderungen unter den Bedingungen ,umfassender Globalisierung“, in: Günther Hellmann/Daniel Jacobi/Ursula Stark Urrestarazu (Hrsg.), "Früher, entschiedener und substanzieller"? Die neue Debatte über Deutschlands Außenpolitik (Zeitschrift für Außen- und Sicherheitspolitik 8 (1)), 379-394.

Messner, Dirk/Guarín, Alejandro/Haun, Daniel, 2013: The Behavioural Dimensions of International Cooperation, Global Cooperation Research Papers No.1, Käte Hamburger Kolleg/Centre for Global Cooperation Research, Duisburg. 
Was folgt auf die Millenniums-Entwicklungsziele?

Messner, Dirk/Nuscheler, Franz, 1998: Die Weltkonferenzen der 1990er Jahre, Bonn.

Messner, Dirk/Rahmstorf, Stefan, 2010: Tipping Points in the Earth System and their Implications for World Politics and for the World Economy, in: Dirk Messner u. a. (Hrsg.), Global Trends 2010: Peace - Development - Environment, Bonn, 63-80.

$O W G$, 2014: Open Working Group Proposal for Sustainable Development Goals: http://sustainabledevelopment.un.org/sdgsproposal (Stand: 02.02.15).

Raworth, Kate, 2012: A Safe and Just Space for Humanity. Can We Live Within the Doughnut?, Oxford.

Raworth, Kate, 2014: Will these Sustainable Development Goals get us into the Doughnut (aka a safe and just Space for Humanity)?, http://oxfamblogs.org/ fp2p/will-these-sustainable-development-goals-get-us-into-the-doughnut-akaa-safe-and-just-space-for-humanity-guest-post-from-kate-raworth/ (Stand: 02.02.15).

Rockström, Johan/Sachs, Jeffrey D./Öhman, Marcus. C./Schmidt-Traub, Guido, 2013: Sustainable Development and Planetary Boundaries, Background Research Paper Submitted to the High Level Panel on the Post-2015 Development Agenda.

Rockström, Johan/Steffen, Will./Noone, Kevin/Persson, Asa/Chapin, F. Stuart/ Lambin, Eric/Lenton, Timothy M. u. a., 2009 a: Planetary Boundaries: Exploring the Safe Operating Space for Humanity, in: Ecology and Society 14 (2), 32.

Rockström, Johan/Steffen, Will/Noone, Kevin/Persson, Äsa/Chapin, F. Stuart/ Lambin, Eric F./Lenton, Timothy M. u. a., 2009 b: A Safe Operating Space for Humanity, in: Nature 46: 472-475.

Steffen, Will/Persson, Åsa/Deutsch, Lisa/Zalasiewicz, Jan/Williams, Mark/ Richardson, Katherine/Crumley, Carole u. a., 2011: The Anthropocene: From Global Change to Planetary Stewardship, in: Ambio 40: 739-761.

Steffen, Will/Richardson, Katherine/Rockström, Johan/Cornell, Sarah E./Fetzer, Ingo/Bennett, Elena M./Biggs, R./Carpenter, Stephen R./de Vries, Wim/de Wit, Cynthia A./Folke, Carl/Gerten, Dieter/Heinke, Jens/Mace, Georgina M./Persson, Linn M./Ramanathan, Veerabhadran/Reyers, B./Sörlin, Sverker, 2015: Planetary Boundaries: Guiding Human Development on a Changing Planet, in: Science (online first).

Walker, Mike/Johnsen, Sigfus/Rasmussen, Sune Olander/Popp, Trevor/Steffensen, Jørgen-Peder/Gibbard, Phil/Hoek, Wim/Lowe, John/Andrews, John/Björck, Svante/Cwynar, Les C./Hughen, Konrad/Kershaw, Peter/Kromer, Bernd/Litt, 
Thomas/Lowe, David J./Nakagawa, Takeshi/Newnham, Rewi/Schwander, Jacob, 2009: Formal Definition and Dating of the GSSP (Global Stratotype Section and Point) for the Base of the Holocene using the Greenland NGRIP Ice Core, and Selected Auxiliary Records, in: Journal of Quaternary Science 24 (1), 3-17.

$W B G U, 1995$ : Szenario zur Ableitung globaler CO2-Reduktionsziele und Umsetzungsstrategien. Stellungnahme zur ersten Vertragsstaatenkonferenz der Klimarahmenkonvention in Berlin. Sondergutachten, Wissenschaftlicher Beirat für Globale Umweltveränderungen (WBGU), Berlin.

$W B G U$, 2011: Welt im Wandel - Gesellschaftsvertrag für eine Große Transformation. Hauptgutachten, Wissenschaftlicher Beirat für Globale Umweltveränderungen (WBGU), Berlin.

$W B G U$, 2014: „Zivilisatorischer Fortschritt innerhalb planetarischer Leitplanken - Ein Beitrag zur SDG-Debatte", Wissenschaftlicher Beirat für Globale Umweltveränderungen (WBGU), Berlin.

Korrespondenzanschrift:

Dr. Clara Brandi

German Development Institute / Deutsches Institut für Entwicklungspolitik (DIE) Tulpenfeld 6

53113 Bonn

E-Mail: Clara.Brandi@die-gdi.de

Prof. Dr. Dirk Messner

Direktor - German Development Institute / Deutsches Institut für Entwicklungspolitik (DIE)

Tulpenfeld 6

53113 Bonn

E-Mail: Dirk.Messner@die-gdi.de 